\title{
Generalized Field Propagator for Arbitrary Finite-Size Photonic Band Gap Structures
}

\author{
Olivier J. F. Martin* \\ Electromagnetic Fields and Microwave Electronics Laboratory, Swiss Federal Institute of Technology, ETH-Zentrum, \\ 8092 Zurich, Switzerland \\ Christian Girard \\ Centre d'Elaboration de Matériaux et Etudes Structurales, CEMES/CNRS, B.P. 4347, 31055 Toulouse Cedex 4, France \\ David R. Smith and Sheldon Schultz \\ Department of Physics, University of California, San Diego, 9500 Gilman Drive, La Jolla, California 92093-0319
}

(Received 23 June 1998)

\begin{abstract}
We investigate the properties of photonic band gap structures of finite size and arbitrary geometry using the density of states deduced from scattering calculations. We first demonstrate this procedure on a finite $2 \mathrm{D}$ array of cylinders and then study at optical frequencies a system formed by a finite array of finite height cylinders positioned on a substrate and illuminated with an evanescent field. [S0031-9007(98)08191-5]
\end{abstract}

PACS numbers: 42.70.Qs, 42.25.Fx, 42.68.Mj

The electromagnetic (EM) properties of infinitely periodic dielectric and metallic systems have been studied extensively in the context of photonic band gap (PBG) structures [1,2], where powerful numerical methods exist that take advantage of the periodicity. Any physical realization of a PBG structure, however, is finite, and thus will have electromagnetic properties and phenomena distinct from an infinite structure. Such phenomena may include surface modes, sensitivity to boundary termination, or band-edge resonances [3-5].

The objective of this Letter is twofold. First, we present a new method of computing the EM properties of a finite PBG structure. Second, we use this approach to study an array of finite-height dielectric cylinders on a substrate, illuminated with an evanescent field. This geometry was chosen to demonstrate the versatility of the scattering method, since it is a nontrivial three-dimensional finite system with open boundary conditions. Furthermore, this configuration is accessible experimentally, requiring only a single PBG layer which could readily be fabricated by lithographic methods.

Accurate numerical methods previously reported for calculating the properties of finite PBG structures include modal method [6], finite difference time domain [7], transfer matrix [8,9], and repeated supercell [10].

Our method uses a scattering solution to obtain the EM modes and modes density associated with an arbitrary finite PBG region. The scattering solution is based on the Green's tensor technique in the frequency domain.

Let us consider a scattering system $\varepsilon(\mathbf{r} ; \omega)$ embedded in an infinite homogeneous background $\varepsilon_{\mathrm{B}}(\omega)$ like the finite $(n \times m)$ lattice of infinite cylinders shown in Fig. 1(a). We assume harmonic fields with the usual $\exp [-i \omega t]$ dependence. When the system is illuminated by an incident field $\mathbf{E}^{0}(\mathbf{r} ; \omega)$, the total electric field
$\mathbf{E}(\mathbf{r} ; \omega)$ is a solution of the Fredholm equation of the second kind,

$$
\begin{aligned}
\mathbf{E}(\mathbf{r} ; \omega)= & \mathbf{E}^{0}(\mathbf{r} ; \omega) \\
& +\int d \mathbf{r}^{\prime} \mathbf{G}^{0}\left(\mathbf{r}, \mathbf{r}^{\prime} ; \omega\right) \cdot \frac{\omega^{2}}{c^{2}} \Delta \varepsilon\left(\mathbf{r}^{\prime} ; \omega\right) \mathbf{E}\left(\mathbf{r}^{\prime} ; \omega\right),
\end{aligned}
$$

where $c$ is the speed of light; $\Delta \varepsilon$ is the dielectric contrast: $\Delta \varepsilon(\mathbf{r} ; \omega)=\varepsilon(\mathbf{r} ; \omega)-\varepsilon_{\mathrm{B}}(\omega)$; and the integration runs over the entire scatterer volume. The kernel $\mathbf{G}^{0}$ of Eq. (1) is the Green's tensor associated with an infinite homogeneous background $\varepsilon_{\mathrm{B}}$. The value of this dyadic for $2 \mathrm{D}$ and $3 \mathrm{D}$ systems is given in Ref. [11]. Since $\mathbf{G}^{0}$
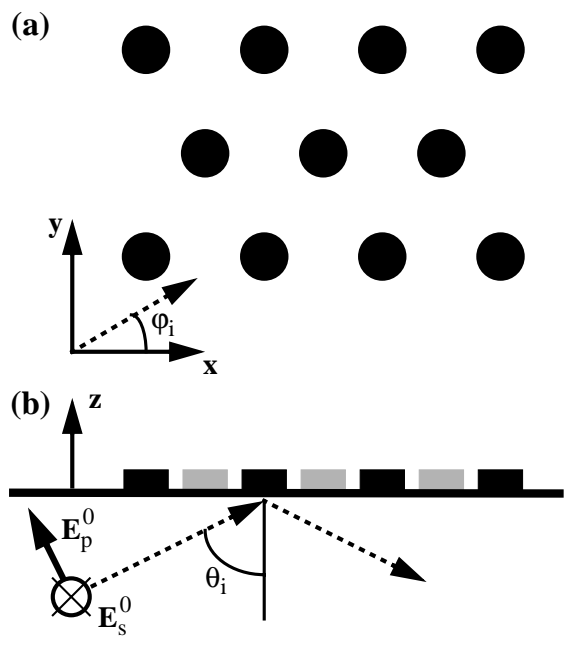

FIG. 1. Geometry of the system under study. (a) Cross section of a finite size $(4 \times 3) 2 \mathrm{D}$ array of infinite dielectric cylinders on a triangular lattice; (b) surface structure composed by the same array but with finite-height cylinders deposited on a transparent substrate and illuminated from underneath by total internal reflection. 
diverges for $\mathbf{r}=\mathbf{r}^{\prime}$ the principal value must be taken in Eq. (1) as described in Ref. [11].

For simplicity, let us rewrite Eq. (1) in operator notation,

$$
\mathbf{E}=\mathbf{E}^{0}+\mathbf{G}^{0} \cdot \Delta \varepsilon \mathbf{E} .
$$

We recently showed that an alternative form of Eq. (2) existed [12],

$$
\mathbf{E}=\mathbf{E}^{0}+\mathbf{G} \cdot \Delta \varepsilon \mathbf{E}^{0},
$$

where the Green's tensor G fulfills Dyson's equation,

$$
\mathbf{G}=\mathbf{G}^{0}+\mathbf{G}^{0} \cdot \Delta \varepsilon \mathbf{G},
$$

and forms the basis of the generalized field propagator formalism introduced in Ref. [12]. The tensor $\mathbf{G}$ contains the entire response of the system under study and provides a powerful way of computing the scattered field for arbitrary excitations.

To investigate finite-size PBG structures, one can use the previous formalism to compute the field transmitted or reflected by the structure as a function of the frequency and identify allowed or forbidden frequency regions from these data. Alternatively, however, we can compute the density of states (DOS) directly from the Green's tensor $\mathbf{G}$, which also functions as an important indicator of band gap regions in PBG structures.

In analogy with quantum mechanics [13], the DOS is obtained from the imaginary part of the trace of the Green's tensor $\mathbf{G}$,

$$
\operatorname{DOS} \sim \operatorname{Im}\{\omega \operatorname{Tr}(\mathbf{G})\} .
$$

It is important to note that the numerical DOS obtained this way is related to the space spanned by the discretized geometry used for the calculation of G. Note that one can also define a local density of states $\operatorname{DOS}(\mathbf{r}) \sim$ $\operatorname{Im}\{\omega \mathbf{G}(\mathbf{r}, \mathbf{r})\}$ which accounts for the variation of spontaneous emission at a particular location [14].

To demonstrate the correlation of the DOS with the band gap regions of a finite PBG structure, we consider a finite $(6 \times 6)$ array of cylinders with radius $r$, placed on a triangular lattice of period $a$ [Fig. 1(a)]. We assume that the cylinders are infinitely long and the field propagates in the plane of Fig. 1(a). Two different polarization modes exist in such a 2D system: Transverse magnetic (TM), where the electric field is parallel to the cylinders axis, and transverse electric (TE), where it is in the plane of Fig. 1(a).

The TM photonic band structure, computed using the plane wave method [15], for an infinitely periodic lattice is given in the left part of Fig. 2. Four different forbidden regions can be observed. In the right part of Fig. 2 we present the DOS computed with Eq. $(5)$ for a $(6 \times 6)$ finite array. The four different forbidden regions are also clearly evident from these data. Typically, peaks occur in the DOS at the band edge, as has also been observed for an infinite triangular lattice [16]. Let us emphasize that the DOS shown in Fig. 2 is computed for a geometry with extremely limited extent; all together, there are only 33 cylinders in the system.

To observe band gaps for TE modes, we reduce the filling factor to $r / a=0.2$ [17], leading to a PBG around $\omega=2 \pi c / a$. In Fig. 3 we compare the TE band structure for an infinite lattice with the DOS computed for a finite $(6 \times 6)$ array. Again, there is excellent agreement between the two.

It seems appropriate here to say a few words on the practical calculation of DOS for TM and TE polarizations using Eq. (5). For TM polarization, the electric field has only one component, $\mathbf{E}=E_{z} \mathbf{e}_{z}$ (Fig. 1). Green's tensors $\mathbf{G}^{0}$ and $\mathbf{G}$ reduce then to scalar functions. On the other hand, for TE polarization, the electric field has two components: $\mathbf{E}=E_{x} \mathbf{e}_{x}+E_{y} \mathbf{e}_{y}$ so that $\mathbf{G}^{0}$ and $\mathbf{G}$ become $(2 \times 2)$ matrices [11]. For each polarization, $\mathbf{G}$ can be computed from $\mathbf{G}^{0}$ using an iterative series of Dyson's equations [12] and then DOS obtained by summing the diagonal elements of $\mathbf{G}$.

We now present a finite-size PBG structure similar to that investigated in Fig. 3, but which is amenable to fabrication for use at optical wavelengths.

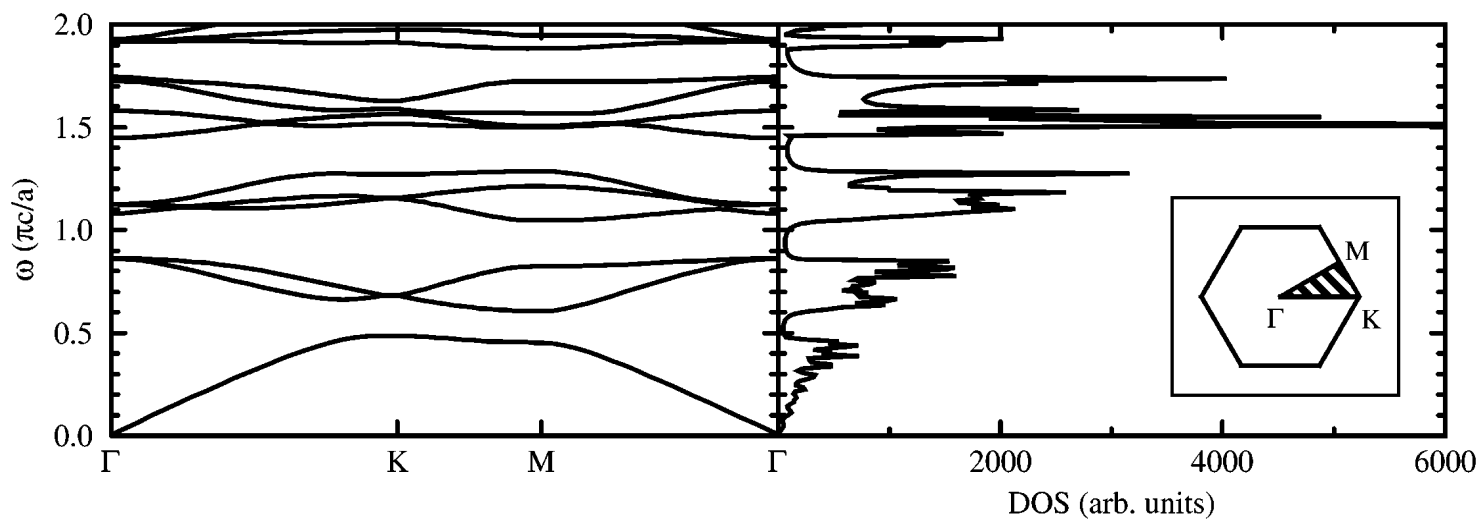

FIG. 2. 2D PBG structure of infinitely long dielectric cylinders $(\varepsilon=9)$ in air $\left(\varepsilon_{\mathrm{B}}=1\right)$ on a triangular lattice with a filling factor $r / a=0.35$ and for TM polarization; the corresponding irreducible Brillouin zone is shown in the inset. Left: Band structure computed for an infinite lattice using plane wave expansion; right: DOS for a finite $(6 \times 6)$ lattice computed with Eq. (5). Note the clear correlation between the band gaps (left) and the minima in DOS (right). 


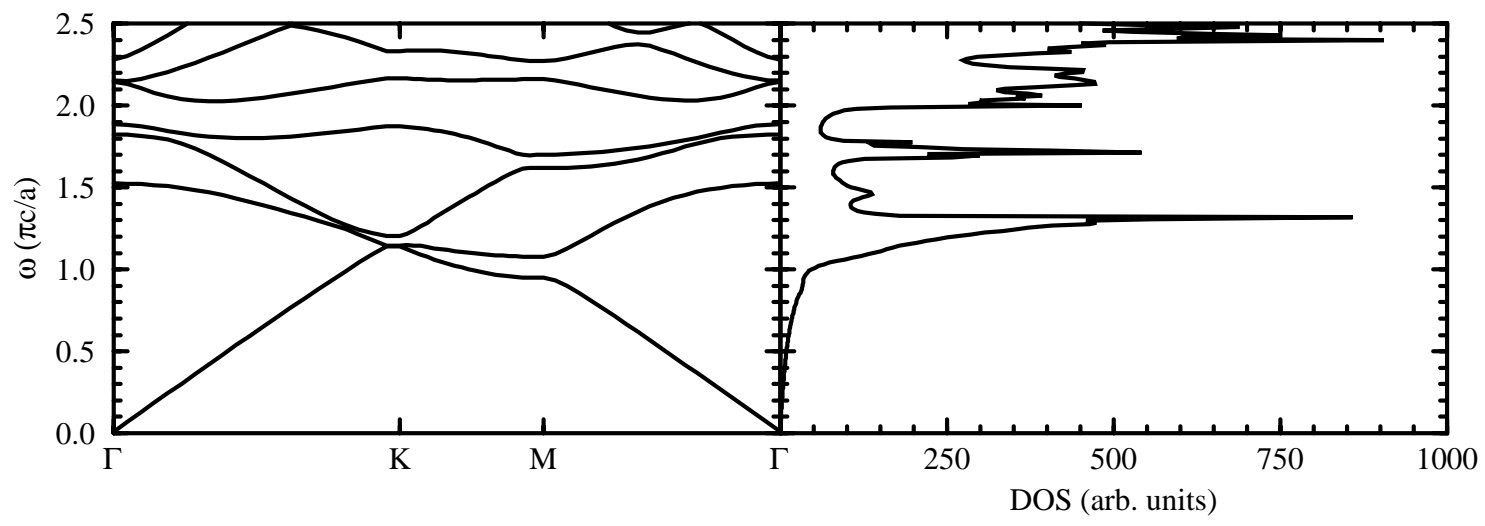

FIG. 3. Same situation as in Fig. 2, but for TE polarization and a filling factor $r / a=0.2$.

Consider a dielectric substrate patterned with an $(6 \times$ 6) array of finite-height cylinders [Fig. 1(b)], illuminated from underneath at a large angle of incidence $\theta_{i}$, so that the field is totally internally reflected (TIR). This illumination mode generates an evanescent field above the substrate that propagates in the direction parallel to the surface.

From the two $s$ and $p$ illumination polarizations that can be distinguished in this system [Fig. 1(b)], we will only consider here $s$ polarization since it corresponds to the TE polarization of a 2D system. Indeed, for $s$ polarization the incident field is parallel (and therefore continuous) to all horizontal interfaces, including at the ends of the finiteheight cylinders. Therefore, such a 3D system exhibits behavior similar to a $2 \mathrm{D}$ system under TE polarization.

For given permittivities $\varepsilon$ and $\varepsilon_{\mathrm{B}}$, the location of the PBG depends only on the filling factor $r / a$. It is therefore possible, within experimentally realistic limits, to scale the geometry so that a PBG arises in a particular frequency region. We take cylinders with radius $r=120 \mathrm{~nm}$ and height $h=80 \mathrm{~nm}$ on a triangular lattice of period $a=$ $600 \mathrm{~nm}$. Such dimensions are within the capability of electron beam lithography or even UV contact lithography [18]. The permittivity of the cylinders is $\varepsilon=9$, so that this system is similar to the structure described in Fig. 3, with the frequency $\omega=2 \pi c / a$ corresponding to a wavelength $\lambda=600 \mathrm{~nm}$. For the substrate we take $\varepsilon_{\text {sub }}=2$ which gives a TIR critical angle $\theta_{\mathrm{i}}=45^{\circ}$. To ensure that only an evanescent field is established above the substrate, we calculate for a larger angle and take $\theta_{\mathrm{i}}=70^{\circ}$. The incident field is propagating at an angle $\varphi_{\mathrm{i}}=35^{\circ}$ through the lattice [Fig. 1(a)].

In Fig. 4 we present the field intensity above this finitesize array of cylinders as a function of the illumination wavelength $\lambda$ (for convenience a frequency scale similar to that of Fig. 3 is also shown). The corresponding surface Green's tensor $\mathbf{G}^{0}$ is given in Ref. [19]. The extremely sharp peak at $\lambda=693 \mathrm{~nm}$ corresponds to the DOS peak at the lower band edge in Fig. 3. The band gap region between $\lambda \approx 600-700 \mathrm{~nm}[\omega \approx(2-1.7)(\pi c / a)]$ is clearly demonstrated in Fig. 4 . The behavior of the transmitted field above the substrate is quite complex and will be presented in detail elsewhere.

The intensity sharp peak at $\lambda=693 \mathrm{~nm}$ corresponds to extremely strong scattering by the structure, as illustrated in Fig. 5(a), where we present the field intensity distribution above the system. Note that the field is scattered in a specific geometrical pattern in the region outside of the actual array. The maximum intensity in this figure is 9.0 times the intensity of the illuminating field below the surface, which is particularly striking when one recalls that for TIR illumination only an evanescent field is established above the surface. The intensity measured in Fig. 5(a) therefore corresponds to an enhancement of $2.7 \times 10^{4}$ compared to the field intensity that would be observed above a bare surface.

A completely different field distribution is observed at another wavelength, e.g., $\lambda=600 \mathrm{~nm}$ [Fig. 5(b)]. There the field remains strongly localized in the region of the cylinder array and now reaches an intensity 1.3 times that

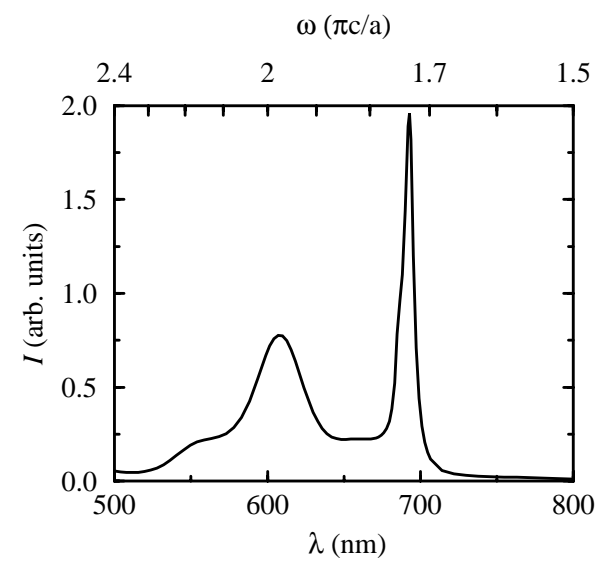

FIG. 4. Field intensity above a $(6 \times 6)$ finite-size surface structure [Fig. 1(b)], as a function of the illuminating wavelength. Same lattice parameters as in Fig.3. The total field intensity $I$ is computed at a constant height $z=500 \mathrm{~nm}$ above the substrate. The intensity of the illuminating field below the surface (TIR) is equal to 1 . 

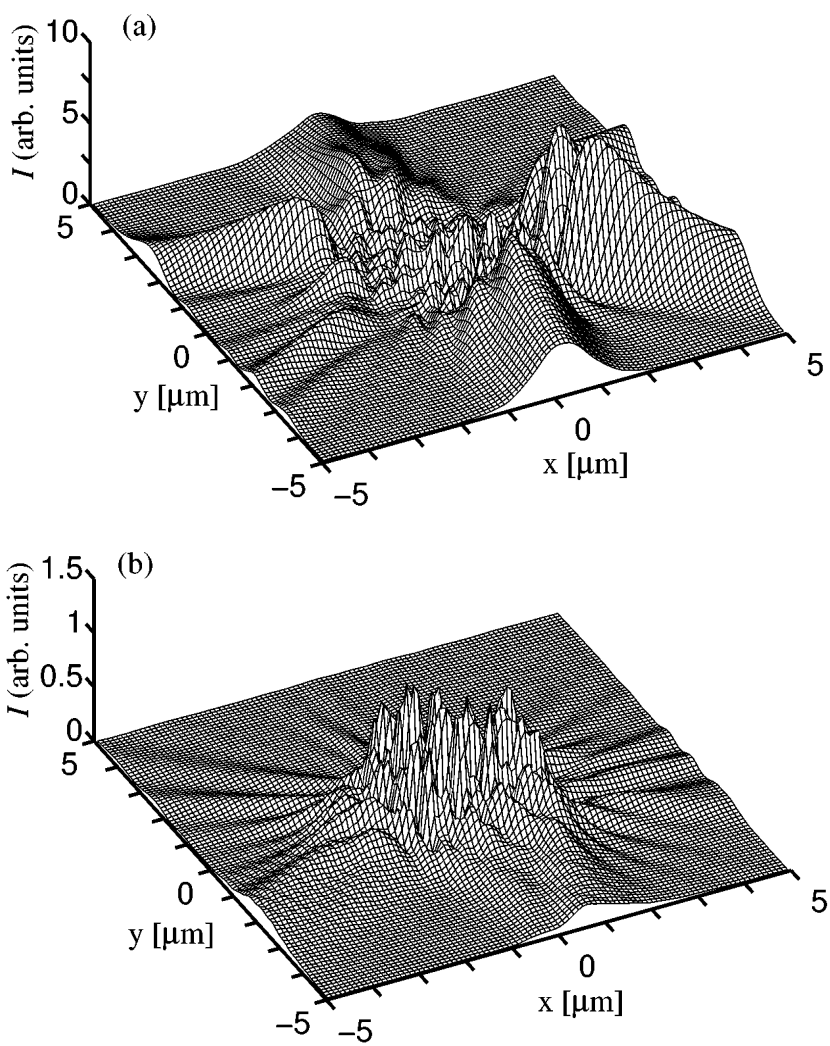

FIG. 5. Topology of the field intensity above a $(6 \times 6)$ finitesize surface structure [Fig. 1(b)]. The intensity is computed at a constant height $z=500 \mathrm{~nm}$ above the substrate for two different illuminating wavelengths: (a) $\lambda=693 \mathrm{~nm}$ and (b) $\lambda=600 \mathrm{~nm}$.

incident, which again has to be compared to the evanescent value of the order of $10^{-4}$.

This strong scattered field remains bound to the surface and rapidly decreases when one moves away from the interface.

Contrary to the extraordinary optical transmission measured recently by Ebbesen et al. on subwavelength hole arrays in metallic films [20] or to former reflection experiments by Kitson et al. [21], the strong scattering enhancement evidenced in our system does not involve any plasmon resonances, and incorporates only a very limited number of scatterers.

We believe that finite surface PBG structures under TIR illumination are attractive from several points of view: They constitute open 2D systems, where the topology of the transmitted field can be measured, e.g., by means of optical near-field microscopy [22]. Since the system is open, active components, such as fluorescent molecules, can be placed within the lattice to investigate radiative interaction with the greatly altered DOS [23]. The strong enhancement of the field above the surface as well as the marked frequency selectivity may be of use in imaging or integrated optic devices. Finally, this enhanced field could also be used for making evanescent-wave atom mirrors [24].

In addition, the direct calculation of DOS based on the generalized field propagator technique as presented in this Letter should be useful for the investigation of finitesize PBG structures with arbitrary shaped scatterers or for random scattering systems.

This work was supported by the Swiss National Science Foundation and by the U.S. NSF (No. NSF-DMR-9623949).

*E-mail address: martin@ifh.ee.ethz.ch

[1] C. M. Bowden, J. P. Dowling, and H. O. Everitt, J. Opt. Soc. Am. B 10, 280 (1993).

[2] Photonic Band Gaps and Localization, edited by C. M. Soukoulis (Plenum, New York, 1993).

[3] R. D.Meade et al., Phys. Rev. B 44, 10961 (1991).

[4] W. M. Robertson et al., Opt. Lett. 18, 528 (1993).

[5] J. M. Bendickson, J.P. Dowling, and M. Scalora, Phys. Rev. E 53, 4107 (1996).

[6] G. Tayeb and D. Maystre, J. Opt. Soc. Am. A 14, 3323 (1997).

[7] P. R. Villeneuve, S. Fan, and J. D. Joannopoulos, Phys. Rev. B 54, 7837 (1996).

[8] M. Sigalas et al., Phys. Rev. B 48, 14121 (1993).

[9] J. B. Pendry, J. Mod. Opt. 41, 209 (1994).

[10] R. D. Meade et al., Phys. Rev. B 48, 8434 (1993).

[11] O.J.F. Martin and N.B. Piller, Phys. Rev. E 58, 3909 (1998).

[12] O. J. F. Martin, C. Girard, and A. Dereux, Phys. Rev. Lett. 74, 526 (1995).

[13] E. N. Economou, Green's Functions in Quantum Physics (Springer-Verlag, Berlin, 1990), 2nd ed.

[14] F. Wijnands et al., Opt. Quantum Electron. 29, 199 (1997).

[15] D. R. Smith et al., J. Opt. Soc. Am. B 10, 314 (1993).

[16] M. Plihal and A.A. Maradudin, Phys. Rev. B 44, 8565 (1991).

[17] J. N. Winn, R. D. Meade, and J. D. Joannopoulos, J. Mod. Opt. 41, 257 (1994).

[18] H. Schmid et al., Appl. Phys. Lett. 72, 2379 (1998).

[19] C. Girard et al., Phys. Rev. B 52, 2889 (1995).

[20] T. W. Ebbesen et al., Nature (London) 391, 667 (1998).

[21] S. C. Kitson et al., Phys. Rev. Lett. 77, 2670 (1996).

[22] Photons and Local Probes, edited by O. Marti and R. Möller (Kluwer, Dordrecht, The Netherlands, 1995).

[23] E. Yablonovitch, Phys. Rev. Lett. 58, 2059 (1987).

[24] J. P. Dowling and J. Gea-Banacloche, Adv. At. Mol. Opt. Phys. 37, 1 (1996). 